\title{
Meduloepitelioma teratoide maligno. Reporte de caso
}

\section{Malignant teratoid medulloepithelioma. A case report}

\author{
Welsi J. García-Barbosa* y Alfredo Lizárraga-Corona
}

Departamento de Oftalmología, Hospital de Pediatría, Centro Médico Nacional de Occidente, Instituto Mexicano del Seguro Social, Guadalajara, México

\begin{abstract}
Resumen
El meduloepitelioma intraocular es un tumor embrionario poco frecuente. El diagnóstico clínico se establece mediante la presencia de tumor a nivel de cuerpo ciliar, quistes intratumorales confirmados mediante ultrasonido, aunado a características clínicas como neovascularización iridiana, corectopia, ectropión uveal, membrana ciclítica retrolental. La confirmación del diagnóstico se hace mediante estudio histopatológico. El tratamiento estándar es enucleación. Presentamos el caso de un meduloepitelioma teratoide maligno confinado al globo ocular en una paciente de 1 año de edad. Los estudios de extensión fueron negativos, por lo que se mantuvo en vigilancia estrecha por el Servicio de Oftalmología y Servicio de Oncología Pediátrica después de la enucleación. Comparado con otros tumores intraoculares, el meduloepitelioma tiene una fuerte tendencia a producir glaucoma secundario, se ha descrito que el $60 \%$ de estos tumores desarrollarán hipertensión ocular. Esto parece deberse a la neovascularización iridiana. Es importante en pacientes de edad pediátrica que acuden a consulta con un cuadro clínico de buftalmos, además de la sospecha de glaucoma congénito, buscar de manera intencionada la presencia de un tumor intraocular mediante estudios de imagen, y así brindar un tratamiento adecuado y oportuno. Clínica e histológicamente, el diagnóstico diferencial se debe realizar con el retinoblastoma, rabdomiosarcoma, neuroblastoma, malformaciones vasculares, quistes hemáticos, glioneuroma y con la hiperplasia primaria del vítreo. El meduloepitelioma teratoide maligno tiene un riesgo de mortalidad del 10\%, relacionado con la extensión intra y extracraneal (principalmente a ganglios linfáticos regionales).
\end{abstract}

Palabras clave: Meduloepitelioma intraocular. Meduloepitelioma teratoide. Tumor del cuerpo ciliar. Tumor intraocular. Glaucoma.

\begin{abstract}
Intraocular medulloepithelioma is an uncommon embryonic tumor. Its diagnosis is based on the clinical findings such as the presence of a ciliary body tumor, intratumoral cysts confirmed by ultrasound, iris neovascularization, corectopia, uveal ectropion and retrolental membrane. Diagnosis is confirmed by the pathology report. We present the case of a malignant teratoid medulloepithelioma confined to the eyeball in a 1-year-old patient. Metastasis evaluation was negative, so she remained under close ophthalmological and oncological surveillance after enucleation. Compared with other intraocular tumors, medulloepithelioma is more likely to develop secondary glaucoma, a complication that has been described in $60 \%$ of these tumors, probably secondary to iris neovascularization. In pediatric patients who attend consultation due to buphthalmos and congenital glaucoma under evaluation, it is important to exclude intraocular tumors through imaging studies for timely diagnosis and treatment. Retinoblastoma, rhabdomyosarcoma, neuroblastoma, vascular malformations, hematic cysts, glioneuroma and persistent hyperplastic primary vitreous, should be considered in the differential diagnosis. Malignant teratoid meduIloepithelioma has a risk of mortality of 10\%, related to intra and extracranial dissemination (mainly to regional lymph nodes).
\end{abstract}

Key words: Intraocular medulloepithelioma. Teratoid medulloepithelioma. Ciliary body tumor. Intraocular tumor. Glaucoma.

\section{Correspondencia:}

*Welsi J. García-Barbosa

Belisario Domínguez \#1000

C.P. 44660, Guadalajara, Jal., México

E-mail: welsigarcia@gmail.com

Fecha de recepción: 12-06-2018

Fecha de aceptación: 16-08-2018

DOI: 10.24875/RMO.M19000073 0187-4519/@ 2018 Sociedad Mexicana de Oftalmología. Publicado por Permanyer. Éste es un artículo Open Access bajo la licencia CC BY-NC-ND (http://creativecommons.org/licenses/by-nc-nd/4.0/).
Disponible en internet: 03-07-2019 Rev Mex Oftalmol. 2019;94(1):46-50 www.rmo.com mx 


\section{Introducción}

El meduloepitelioma intraocular es un tumor embrionario poco frecuente, que afecta principalmente al cuerpo ciliar, previamente descrito como teratoneuroma ${ }^{1}$. Habitualmente es unilateral y focal, aunque hay casos bilaterales. Puede afectar otros órganos derivados del neuroepitelio, como la retina, el nervio óptico y el iris². En el sistema nervioso central, es generalmente periventricular, con afectación por orden de frecuencia de los lóbulos temporal, parietal, occipital y frontal ${ }^{1}$. Se ha clasificado como teratoide y no teratoide, el meduloepitelioma no teratoide es una proliferación celular de neuroepitelio, y el meduloepitelioma teratoide se distingue por la presencia de elementos heterólogos adicionales, particularmente cartílago, músculo esquelético y tejido encefálico. Basados en el criterio histopatológico, ambos tipos pueden ser benignos o malignos ${ }^{3}$. Los criterios de malignidad son: 1) áreas de células poco diferenciadas semejantes al retinoblastoma, 2) presencia de componente sarcomatoide o anaplásico, 3) invasión a la úvea o esclerótica, 4) pleomorfismo celular, 5) mitosis y 6) invasión extraocular ${ }^{2}$.

El diagnóstico de meduloepitelioma es básicamente histopatológico. En algunos casos, la presencia de mucopolisacáridos es de utilidad para su confirmación. La inmunohistoquímica sirve para la identificación de elementos celulares heterotópicos, y muestra utilidad para identificar neuroepitelio, sobre todo en tumores pobremente diferenciados, en los que no se identifican la formación de rosetas y/o cordones 1 .

En tumores pequeños, la escisión local vía iridociclectomía, aunada a radioterapia, ha sido efectuada con éxito variable, ya que estos casos con frecuencia recurren y requieren una segunda intervención mediante enucleación. Algunos meduloepiteliomas malignos se tratan con braquiterapia en combinación con escisión quirúrgica, sin embargo, una vez que se ha realizado el diagnóstico, el tratamiento estándar es la enucleación. Se requiere la exenteración si el tumor se extiende más allá del globo ocular. Si se trata de un tumor con metástasis, se inicia tratamiento combinado mediante quimioterapia y braquiterapia 4 .

Dos tercios de los meduloepiteliomas son malignos y tienen una tasa de mortalidad del $10 \%$. La causa más frecuente de muerte es secundaria a la extensión intra y extracraneana, esta última es principalmente a ganglios linfáticos regionales².

\section{Presentación del caso}

Presentamos el caso de una paciente femenina de 1 año y 5 meses de edad, que es llevada a consulta

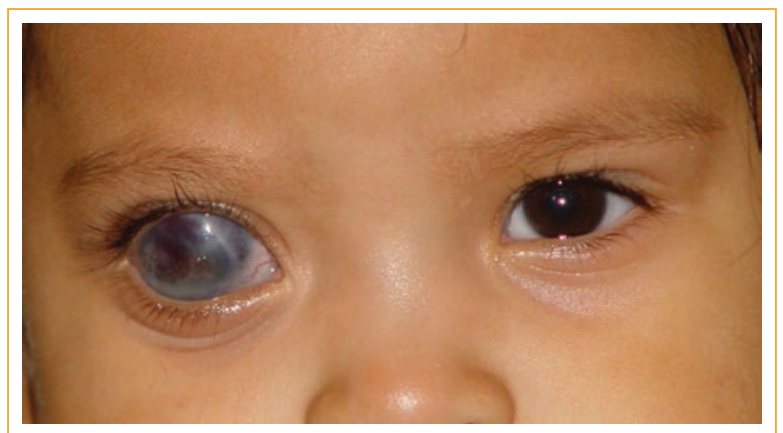

Figura 1. Foto clínica al momento del diagnóstico en su primera consulta.

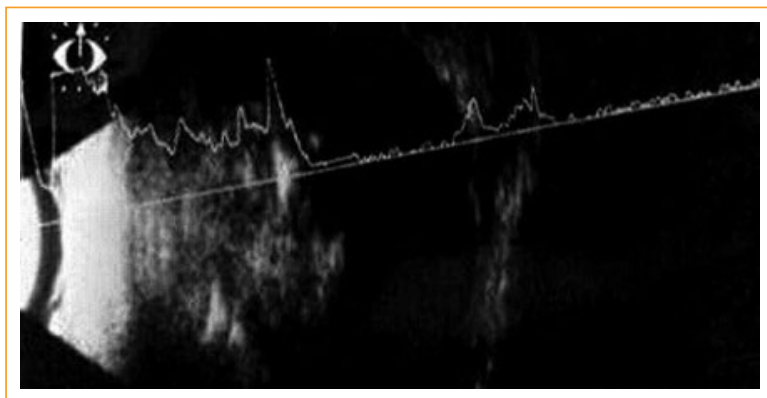

Figura 2. Ultrasonido modo A y B. Globo ocular de contorno normal, zonas densas pequeñas de muy alta reflectividad compatibles con calcificaciones; membranas densas de alta reflectividad, correspondientes a retina desprendida en su totalidad, detrás de las cuales se aprecia una zona quística.

por su madre debido a un notorio aumento de tamaño del globo ocular derecho en los últimos 6 meses, acompañado de irritabilidad. Fue valorada por el Servicio de Oftalmología en el Hospital de Pediatría (HP) del Centro Médico Nacional de Occidente (CMNO), donde se inició abordaje diagnóstico.

En la exploración física encontramos el ojo derecho sin respuesta a estímulo visual, buftalmos, movimientos oculares disminuidos en todas las posiciones de la mirada para el ojo derecho, megalocornea y opacidad corneal, con dificultad para valorar las estructuras del segmento anterior debido a la pérdida de anatomía; fondo de ojo derecho no valorable; ojo izquierdo sin alteraciones (Fig. 1).

El ultrasonido modo A y B del ojo derecho (Fig. 2) reveló un globo ocular de contorno normal, con diámetro anteroposterior de $26.85 \mathrm{~mm}$, cristalino desplazado a la región anterior, zonas densas pequeñas de muy alta reflectividad, compatibles con calcificaciones, membranas densas de alta reflectividad, correspondientes a retina desprendida en su totalidad, detrás de 


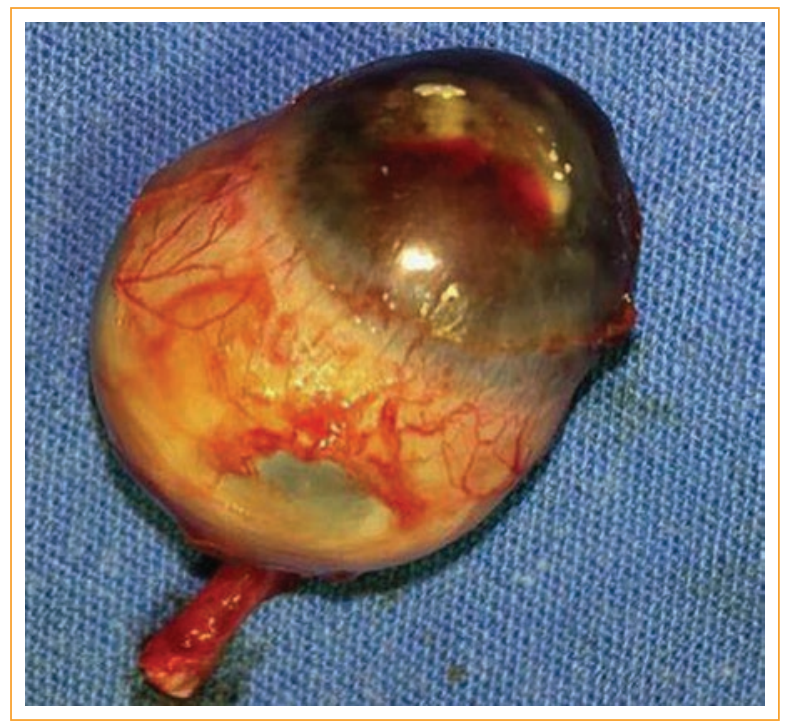

Figura 3. Globo ocular y porción de nervio óptico.

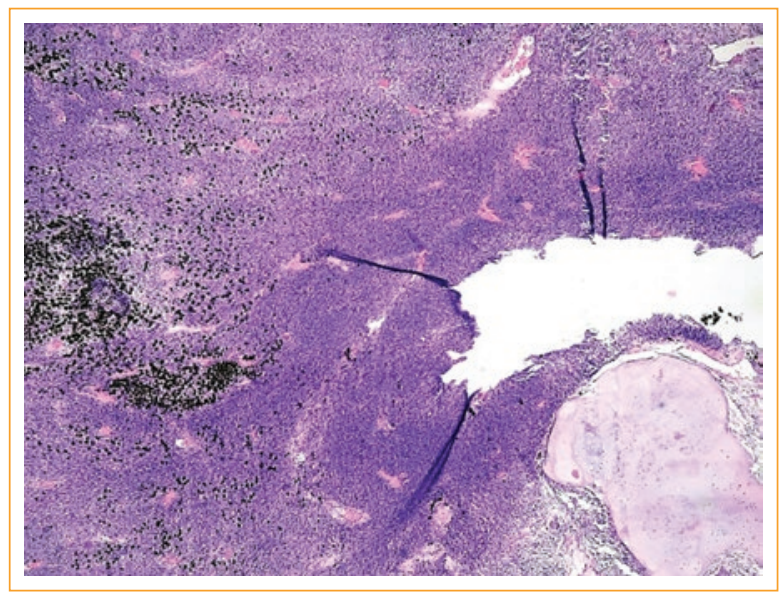

Figura 4. En este campo se observa un área sarcomatosa, que forma un distintivo patrón en fascículos, con celularidad densa. A mayor aumento, se corrobora la presencia de numerosas mitosis y una morfología nuclear fusiforme con escaso citoplasma. Dicho componente se localiza adyacente a una isla de cartílago (inferior derecha) (tinción H-E) (aumento original 40x).

las cuales se aprecia una zona quística, coroides engrosada en $360^{\circ}$.

La resonancia magnética T1 mostró una imagen hiperintensa con involucro de aproximadamente el $50 \%$ del contenido intraocular, sin evidencia de afectación ósea o tejidos blandos adyacentes.

Se realizó enucleación y se envió a estudio histopatológico, que reveló un globo ocular con diámetro anteroposterior de $27 \mathrm{~mm}$ (Fig. 3) y porción de nervio óptico de $15 \mathrm{~mm}$; no se delimita límite corneal; al corte, se observó tumoración, que ocupaba el $65 \%$ del globo ocular, que retrajo la retina,

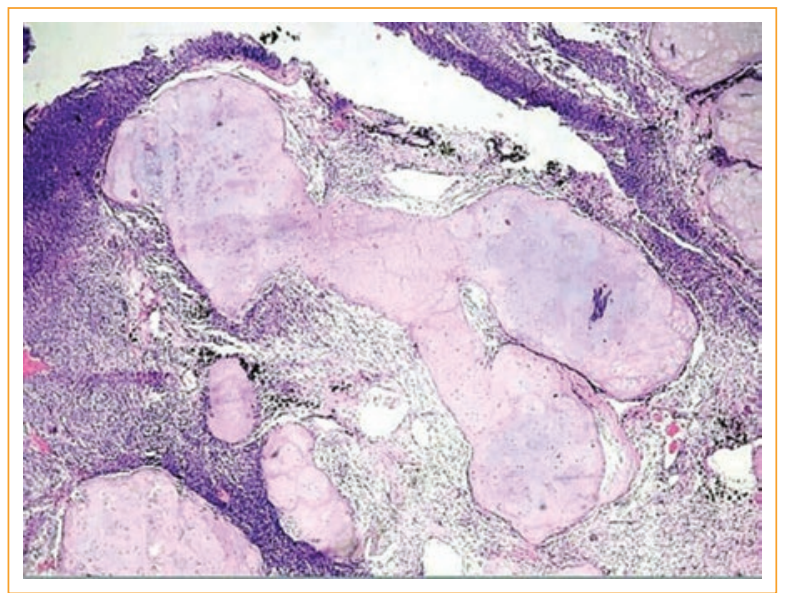

Figura 5. Se observa en la porción central una gran isla de cartílago hialino, rodeada por la misma población celular inmadura. Se repite el patrón histológico (tinción $\mathrm{H}-\mathrm{E})$ (aumento original 40x).

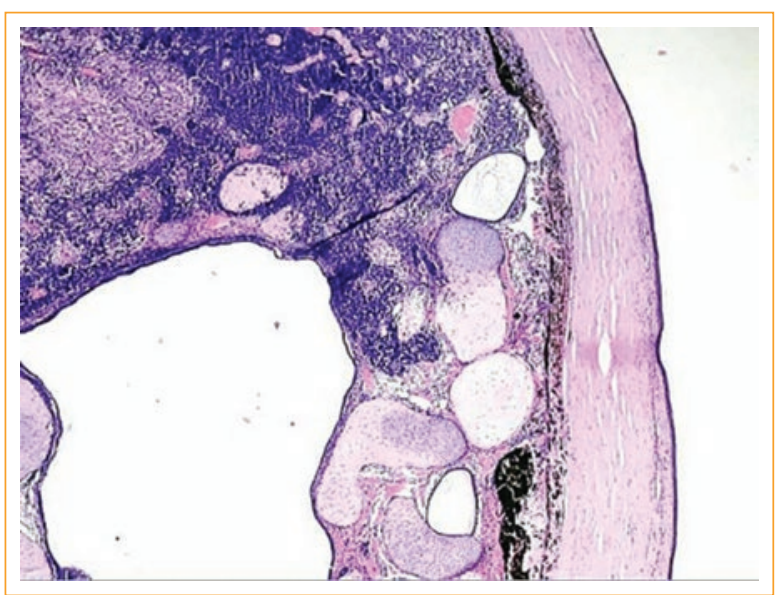

Figura 6. Se aprecian estructuras quísticas con islas de cartílago maduro adyacentes, rodeadas por una población celular densa, intensamente basófila, que corresponde a células de neuroepitelio poco diferenciado. A la derecha se observa la córnea, con tejido uveal adherido (tinción $\mathrm{H}-\mathrm{E}$ ) (aumento original 40x).

localizada en la parte anterior del globo ocular en estrecho contacto con cornea; no se observó arquitectura conservada del cuerpo ciliar e iris. Tumor de $25 \mathrm{~mm}$ de diámetro con bordes moderadamente definidos, coloración blanco grisácea y consistencia heterogénea.

Se identificó al corte histológico meduloepitelioma teratoide maligno, con islotes de cartílago hialino maduro (Figs. 4 y 5), focos de diferenciación neuroblástica poco diferenciados, componente sarcomatoso de células fusiformes y de condrosarcoma; alto índice mitótico. Infiltrando corneal (Fig. 6), tejido uveal, esclerótica 
anterior en sus $2 / 3$ internos y retina. Segmento de nervio óptico sin tumor.

Fue valorada por el Servicio de Oncología Médica del HP del CMNO, que solicitó estudios de extensión tumoral, sin encontrar afectación en el resto de la economía corporal. Al momento, se mantiene en vigilancia por el Servicio de Oftalmología y el Servicio de Oncología Pediátrica del HP del CMNO, sin datos de recidiva a los 18 meses de seguimiento tras diagnóstico.

\section{Discusión}

El meduloepitelioma intraocular es una neoplasia rara típicamente diagnosticada en la primera década de la vida como tumor de cuerpo ciliar $^{3}$, con una edad media de presentación de 5 años ${ }^{5}$. Existen pocos reportes de meduloepitelioma en las pasadas décadas; de una serie de 56 casos reportados de meduloepiteliomas no sindrómicos, la media de presentación fue de 3.8 años, sin predominio de sexo ${ }^{6}$.

El diagnóstico clínico se establece mediante la presencia de tumor a nivel de cuerpo ciliar, quistes intratumorales confirmados mediante ultrasonido, aunado a características clínicas como neovascularización iridiana, corectopía, ectropión uveal, cambios cristalinianos (catarata, luxación de cristalino, coloboma), membrana ciclítica retrolental. La confirmación del diagnóstico es mediante estudio histopatológico ${ }^{5}$.

La baja visual en pacientes con diagnóstico de meduloepitelioma es secundaria a la presencia de membrana neoplásica ciclítica, catarata o subluxación del cristalino. Comparado con otros tumores intraoculares, el meduloepitelioma tiene una fuerte tendencia a producir glaucoma secundario, ya que se ha escrito que el $60 \%$ de estos tumores desarrollaran hipertensión ocular. Parece ser secundario al desarrollo de neovascularización iridiana, por lo tanto, en pacientes de edad pediátrica con glaucoma neovascular y polo posterior normal, es altamente sugestivo de meduloepitelioma en cuerpo ciliar oculto ${ }^{3}$. Dentro del abordaje diagnóstico de nuestra paciente, ante un cuadro clínico de buftalmos y megalocornea, encontramos como uno de los principales diagnósticos diferenciales el glaucoma congénito, sin embargo, al no ser posible la valoración del resto de estructuras intraoculares por opacidad corneal y alteraciones en la anatomía del segmento anterior, fue de vital importancia realizar estudios complementarios para documentar el estado del segmento anterior y posterior del ojo, debido a la probabilidad de encontrarse ante un tumor intraocular. Clínica e histológicamente el diagnóstico diferencial se debe realizar con el retinoblastoma, rabdomiosarcoma, neuroblastoma, malformaciones vasculares, quistes hemáticos, glioneuroma y con la hiperplasia primaria del vítreo ${ }^{7,8}$.

Los hallazgos clínicos que pueden ayudar a distinguir meduloepitelioma de retinoblastoma incluyen: glaucoma (44\%), neovascularización del iris $(51 \%)$, catarata $(20 \%)$, luxación del cristalino $(27 \%)$, coloboma del cristalino $(20 \%)$, y membrana ciclítica retrolental y presencia de quistes intratumorales $(61 \%$ en ambos casos) $)^{6}$.

Una característica clínica sugestiva de meduloepitelioma es la presencia de quistes intratumorales, los cuales están rodeados de neuroepitelio y contienen matriz rica en ácido hialurónico análoga al contenido del vítreo ${ }^{3}$, por lo tanto, si mediante un ultrasonido se encuentra un tumor de alta reflectividad con irregularidad estructural asociado a cambios quísticos que involucran al cuerpo ciliar, se establecerá un diagnóstico presuntivo de meduloepitelioma ${ }^{9}$.

Diversos estudios reportan que, a pesar de tratase de un tumor maligno, no tiende a metastatizar a distancia a menos que se trate de extensión extraocular de la lesión primaria ${ }^{3}$. Se evalúa con estudios de imagen, la posibilidad de dicha afectación. En caso de la paciente, el estudio de resonancia magnética de órbita y cráneo no mostró afectación del sistema nervioso central, por lo que al momento se mantiene en vigilancia conjunta con el Servicio de Oncología Pediátrica.

\section{Conclusiones}

El meduloepitelioma intraocular es un tumor poco frecuente, diagnosticado principalmente en la primera década de la vida. Se presenta con diferentes signos clínicos a nivel del segmento anterior, uno de los principales es la presencia de neovascularización iridiana, que representa una de las principales causas para el desarrollo de glaucoma en pacientes con retraso en el diagnóstico.

Es importante, en pacientes de edad pediátrica que acuden a consulta con cuadro clínico de buftalmos y megalocornea, además de la sospecha de glaucoma congénito, descartar patologías que se asocien con la clínica presentada, y buscar de manera intencionada un tumor intraocular mediante estudios de imagen, y así brindar un tratamiento adecuado y oportuno.

El estudio histopatológico es de vital importancia para decisiones terapéuticas y evaluación pronóstica. El meduloepitelioma teratoide maligno tiene un $10 \%$ de mortalidad, la cual va a depender de la afectación extraocular. En el caso de la paciente, se trató de una 
tumoración confinada al globo ocular que, debido a la poca tendencia a metastatizar, se recomienda únicamente vigilancia estrecha ante la posible recurrencia del tumor o que la afectación sea intra y/o extracraneana, ya que son las principales causas de muerte en este tipo de tumor.

\section{Responsabilidades éticas}

Protección de personas y animales. Los autores declaran que para esta investigación no se han realizado experimentos en seres humanos ni en animales.

Confidencialidad de los datos. Los autores declaran que han seguido los protocolos de su centro de trabajo sobre la publicación de datos de pacientes.

Derecho a la privacidad y consentimiento informado. Los autores han obtenido el consentimiento informado de los pacientes y/o sujetos referidos en el artículo. Este documento obra en poder del autor de correspondencia.

\section{Financiamiento}

Los autores no recibieron patrocinio para llevar a cabo este artículo.

\section{Conflicto de intereses}

Los autores declaran no tener ningún conflicto de intereses.

\section{Bibliografía}

1. Inzulza Barrientos N, Matus Matus G, Torres Marín R. Meduloepitelioma en el adulto. Rev Mex Oftalmol [Internet]. 2014;88(3):128-36. Disponbible en: http://www.sciencedirect.com/science/article/pii/S0187451914000481

2. Danny Soria-Céspedes, Fernando Martínez-Madrigal, Pedro Méndez-Sashida CO-H. Meduloepitelioma pigmentado maligno intraocular. Presentación de dos casos Intraocular malignant pigmented medulloepithelioma. A report of two cases. Rev Esp Patol. 2009;42(3):225-9.

3. Shields JA, Eagle RC, Shields CL, De Potter P. Congenital Neoplasms of the Nonpigmented Ciliary Epithelium (medulloepithelioma). Ophthalmology [Internet]. 1996;103(12):1998-2006. Disponible en: http://linkinghub.elsevier.com/retrieve/pii/S0161642096303941

4. Rao AA, Naheedy JH, Chen JYY, Robbins SL, Ramkumar HL. A clinical update and radiologic review of pediatric orbital and ocular tumors. J Oncol. 2013;2013:1-22

5. Kaliki S, Shields CL, Eagle RC, Vemuganti GK, Almeida A Manjandavida FP, et al. Ciliary body medulloepithelioma: Analysis of 41 cases. Ophthalmology [Internet]. 2013;120(12):2552-9. Disponible en: http://dx.doi.org/10.1016/j.ophtha.2013.05.015

6. Jakobiec FA, Trief D, Rashid A, Rose MF, Minckler D, Vanderveen D, et al. New insights into the development of infantile intraocular meduIloepithelioma. Am J Ophthalmol [Internet]. 2014;158(6):1275-96.e1. Disponible en: http://dx.doi.org/10.1016/j.ajo.2014.08.036

7. Keefe MO, Fulcher T, Kelly P, Lee W, Dudgeon J. Medulloepithelioma of the Optic Nerve Head. Arch Ophthalmol. 2015;115:1325-7.

8. Shields JA, Shields CL, Schwartz RL. Malignant teratoid medulloepithelioma of the ciliary body simulating persistent hyperplastic primary vitreous. Am J Ophthalmol [Internet]. 1989;107(3):296-8. Disponible en: http://dx.doi.org/10.1016/0002-9394(89)90318-8

9. Foster RE, Murray TG, Byrne SF, Hughes JR, Gendron BK, Ehlies FJ, et al. Echographic features of medulloepithelioma. Am J Ophthalmol. 2000;130(3):364-6. 